\title{
CONSTRUÇÃO DE BANCADA EXPERIMENTAL PARA ESTUDO DO COMPORTAMENTO DO TEOR DE UMIDADE RESIDUAL PRESENTE NO MATERIAL RETIDO UTILIZANDO PENEIRA VIBRATÓRIA
}

\author{
F. S. GUERREIRO ${ }^{1 *}$, V. P. BARBOSA ${ }^{1}$, I. K. G. VARGAS ${ }^{1}$, R. GEDRAITE ${ }^{1}$, L. KUNIGK ${ }^{2}$, C. H. \\ ATAÍDE ${ }^{1}$ \\ ${ }^{1}$ Universidade Federal de Uberlândia, Faculdade de Engenharia Química \\ ${ }^{2}$ Centro Universitário do Instituto Mauá de Tecnologia, Campus São Caetano do Sul \\ "e-mail: fernando_guerreiro18@yahoo.com.br
}

\begin{abstract}
RESUMO
Durante o processo de perfuração de poços de petróleo a utilização de fluido de perfuração é de suma importância, devido às diversas funções que ele desempenha, como: estabilização do poço, carreamento do cascalho proveniente do corte das rochas, lubrificação e resfriamento da broca entre muitos outros. Devido a este fato, as características físicas do fluido devem permanecer constantes durante todo o processo, sendo necessário o controle da concentração de sólidos que é feito através de uma unidade de separação sólido líquido. O peneiramento é a primeira etapa utilizada em uma unidade típica de separação sólido-líquido, seguida posteriormente de bateria de hidrocicliones e centrífugas decantadoras. Este trabalho visa o estudo relacionado à primeira etapa, através da utilização da peneira vibratória e o levantamento da influência do fator adimensional força-g sobre o teor de umidade arrastado juntamente com os sólidos separados em protótipo de peneira vibratória, equipado com tela de 175 mesh. além da apresentação da instrumentação e sistema de controle empregados na bancada experimental. Os resultados demonstraram que o aumento do valor do adimensional força-g contribuiu para a diminuição da umidade residual do sólido retido pela peneira, os ensaios também demonstraram que o controle agiu de forma satisfatória durante o processo, sendo capaz de manter a força-g constante mesmo com as variações de quantidade de massa sob a peneira.
\end{abstract}

\section{INTRODUÇÃO}

A utilização de sistemas eletrônicos digitais em controle e automação de processos se iniciou, praticamente, com o próprio advento dos computadores digitais. Já no começo dos anos 60, instalações industriais introduziram o computador como importante dispositivo para controle. Durante aproximadamente 20 anos, tais aplicações concentraram-se na concepção de controle centralizado, ou seja, com o computador reunindo em um único processador eventualmente redundante - todas as suas tarefas (Garcia, 2005; Seborg et al, 2005; Ogata, 2005).

Atualmente, os modernos sistemas digitais de controle distribuídos, apresentam o recurso da distribuição do processamento das informações, viabilizada pelo baixo custo dos microprocessadores, permitindo assim uma confiabilidade similar àquela obtida pelos pioneiros sistemas analógicos convencionais, ao mesmo tempo em que se oferecia toda a 
potencialidade dos sistemas digitais, especialmente sua flexibilidade, conferida pelas funções reprogramáveis. A estes sistemas, juntaram-se desenvolvimentos importantes em controle sequencial e combinatório (intertravamentos), dispondo-se de equipamentos designados como controladores lógicos programáveis (CLP) (Ronaes et al, 2009).

O sistema de controle de sólidos é de suma importância na operação de perfuração de poços de petróleo, sob o ponto de vista operacional, ambiental e econômico. Este fluido possui sólidos em suspensão que têm origem na ação de corte das formações rochosas pela broca de perfuração ou em sua formulação inicial, sendo classificados, de acordo com os seus componentes base, em fluidos aquosos e não aquosos. As propriedades físicas deste fluido devem ser monitoradas e corrigidas para carrear os cascalhos até a superfície; manter a estabilidade mecânica do poço; resfriar a broca; transmitir força hidráulica até a broca; manter os cascalhos em suspensão quando sem circulação; lubrificar a coluna de perfuração, entre outros. Os equipamentos utilizados em uma unidade típica de controle de sólidos para a separação sólido-líquido são divididos em três grupos sequenciais: peneiras vibratórias, bateria de hidrociclones (desareiadores e dessiltadores) e centrífuga decantadora (Lobato et al, 2011).

Um dos métodos mais empregados para remoção de sólidos presentes no fluido de perfuração é fazer o mesmo escoar através de uma tela em vibração. Partículas menores que a abertura da tela passarão juntamente com o fluido. Partículas maiores serão separadas e encaminhadas para possível secagem e posterior descarte. O propósito da vibração é o transporte do cascalho para fora da tela e o aumento da capacidade de processamento de líquido. Esta ação causa uma rápida separação, reduzindo a quantidade de líquido perdido com os sólidos (Raja et al, 2012; Guerreiro et al, 2013).

Os objetivos deste trabalho são avaliar experimentalmente a influência do adimensional força-g sobre o teor de umidade arrastado juntamente com os sólidos separados em protótipo de peneira vibratória equipado com tela de 175 mesh e apresentar a instrumentação empregada na bancada experimental.

\section{MATERIAIS E MÉTODOS}

Foi utilizada como base para o desenvolvimento dos modelos apresentados neste trabalho a unidade experimental mostrada na Figura 1, a qual é formada por uma peneira vibratória com $1,65 \mathrm{~m}$ de comprimento, 0,81 $\mathrm{m}$ de largura e $1,0 \mathrm{~m}$ de altura, equipada com dois motovibradores e uma tela de separação, podendo esta última ser trocada quando necessário; um tanque de 500 L com agitação para alimentação da suspensão à peneira; um tanque de descarga de $200 \mathrm{~L}$ para coleta do material passante e uma bomba centrífuga para transportar este líquido passante de volta ao tanque de alimentação.

A peneira está equipada com dois motovibradores com $0,75 \mathrm{cv}$ de potência cada um. Esses foram montados de maneira a gerar movimentos em sentidos opostos de forma a garantir a vibração adequada da peneira, impondo movimento linear ao material alimentado sobre a tela de peneiramento. Para controlar a intensidade da vibração imposta ao sistema, ajustou-se a frequência de rotação dos motovibradores por meio de um inversor de frequência da marca WEG modelo CFW700 (Liu; Gao, 2012). 
Figura 1 - Unidade de recuperação de fluido de perfuração

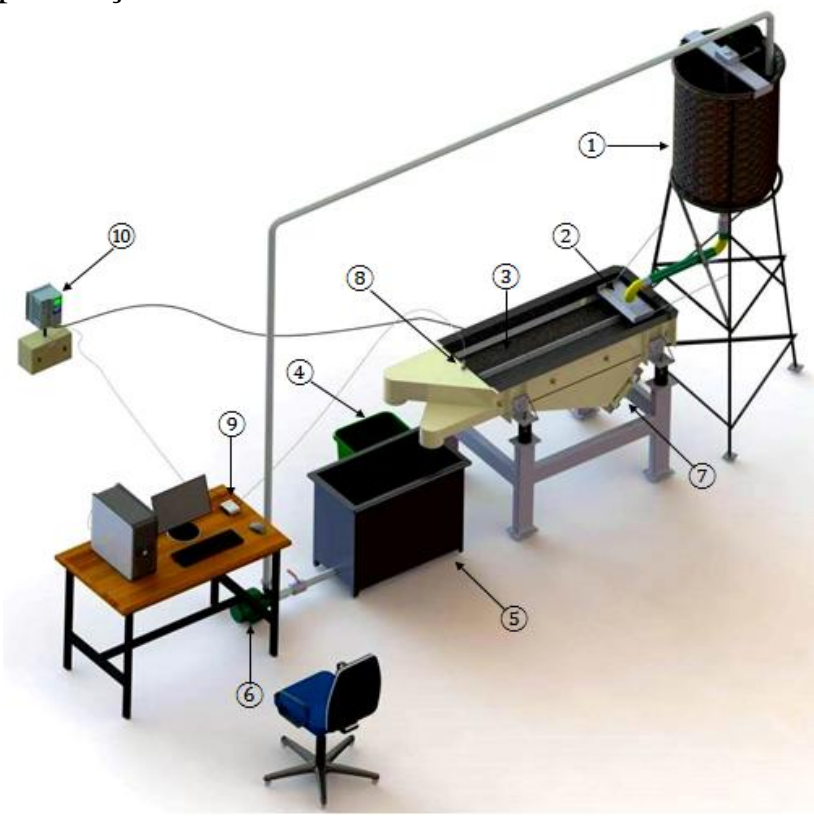

Seus principais componentes são listados a seguir:

1) Tanque de alimentação de 500 litros com agitador;

2) Calha de alimentação;

3) Tela de peneiramento;

4) Balde para coleta do material retido;

5) Tanque de coleta de 200 litros;

6) Bomba centrífuga;

7) Motovibradores;

8) Acelerômetro piezelétrico para medir a vibração da peneira;

9) Placa de aquisição de dados da National Instruments;

10) Inversor de frequência para alterar a rotação dos motovibradores.

Para medir a vibração, foi utilizado um acelerômetro piezelétrico da marca PCB Piezoeletronic modelo 646B00, mostrado na Figura 2, o qual foi responsável por enviar um sinal na faixa de 4 a $20 \mathrm{mAcc}$ a um computador através da placa de aquisição de dados da National Instruments modelo USB 6008 conforme mostra a Figura 3. A grandeza do sinal é dada em força-g numa faixa de 0 a 10 grms.

O programa utilizado para auxiliar a coleta de dados e monitoramento da unidade experimental foi o Labview 2013 da National Instruments, cuja programação é apresentada na Figura 4. Nela, o sinal de corrente gerado pelo acelerômetro era coletado em um taxa da ordem de milisegundos e condicionado em uma faixa de 1 a $5 \mathrm{~V}$. O sistema coletava 1000 valores por segundo. Era calculada a média dos valores coletados para cada intervalo de tempo de 1 segundo e convertida para força-g. Também era possível selecionar um valor de rotação para funcionamento dos motovibradores através do sinal enviado ao inversor de frequência. Para a realização dos experimentos foi necessário estabelecer um valor pré-determinado (set-point) de força-g. Mas à medida que a suspensão era adicionada à peneira, a massa aumentava e, consequentemente, a força-g diminuía. Foi criado um controlador proporcional para compensar este distúrbio. Os dados coletados eram exportados para uma planilha do Excel.

Figura 2 - Acelerômetro usado para aquisição de dados referente à vibração imposta à peneira

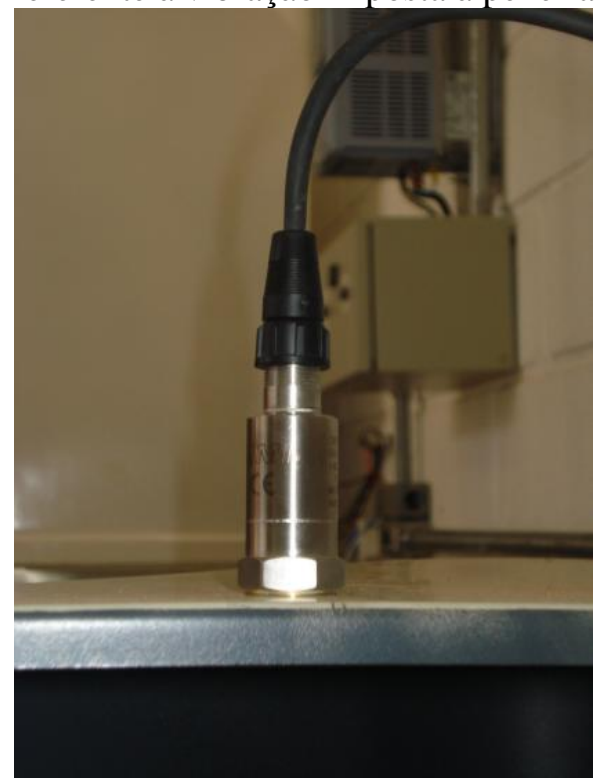


Figura 3 - Placa para aquisição de dados do sinal enviado pelo acelerômetro

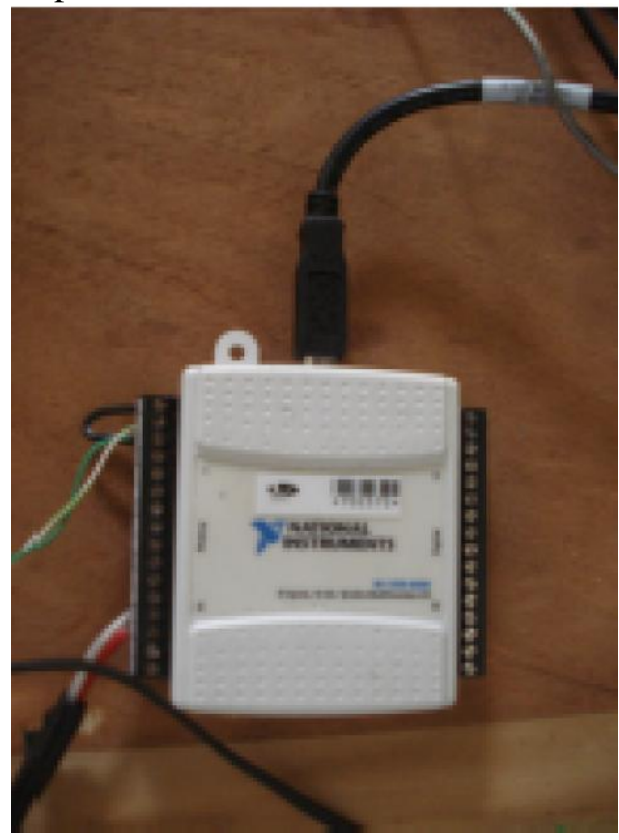

\section{RESULTADOS E DISCUSSÕES}

O comportamento temporal da variável de processo controlada (número adimensional força-G) para a peneira vibratória estudada é apresentada na Figura 5. O valor médio do adimensional de força-g foi de aproximadamente 1,00 e o desvio padrão correspondente foi 0,04 . A variabilidade apresentada pela variável de processo em questão foi inferior a 7,7\%. O comportamento temporal da variável manipulada é apresentado na Figura 6. Com base nas Figuras 5 e 6, pode-se perceber que a malha de controle proposta atuou de maneira coerente e adequada para compensar a tendência de diminuição do valor do adimensional de força-g à medida que o material sólido granular era alimentado sobre a tela da peneira.

Figura 4 - Diagrama de blocos correspondente à malha de controle configurada

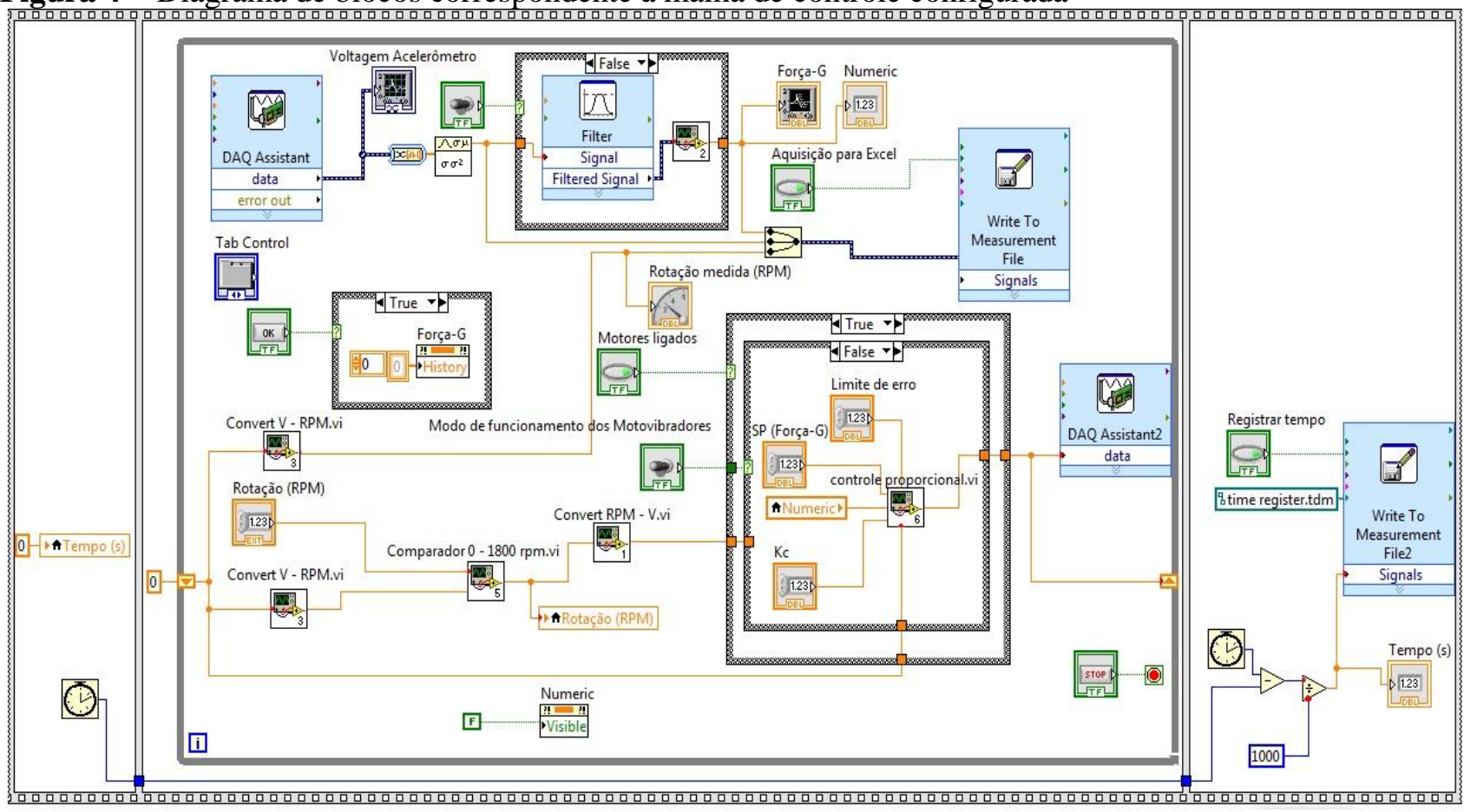


Figura 5 - Comportamento temporal do adimensional de força-g

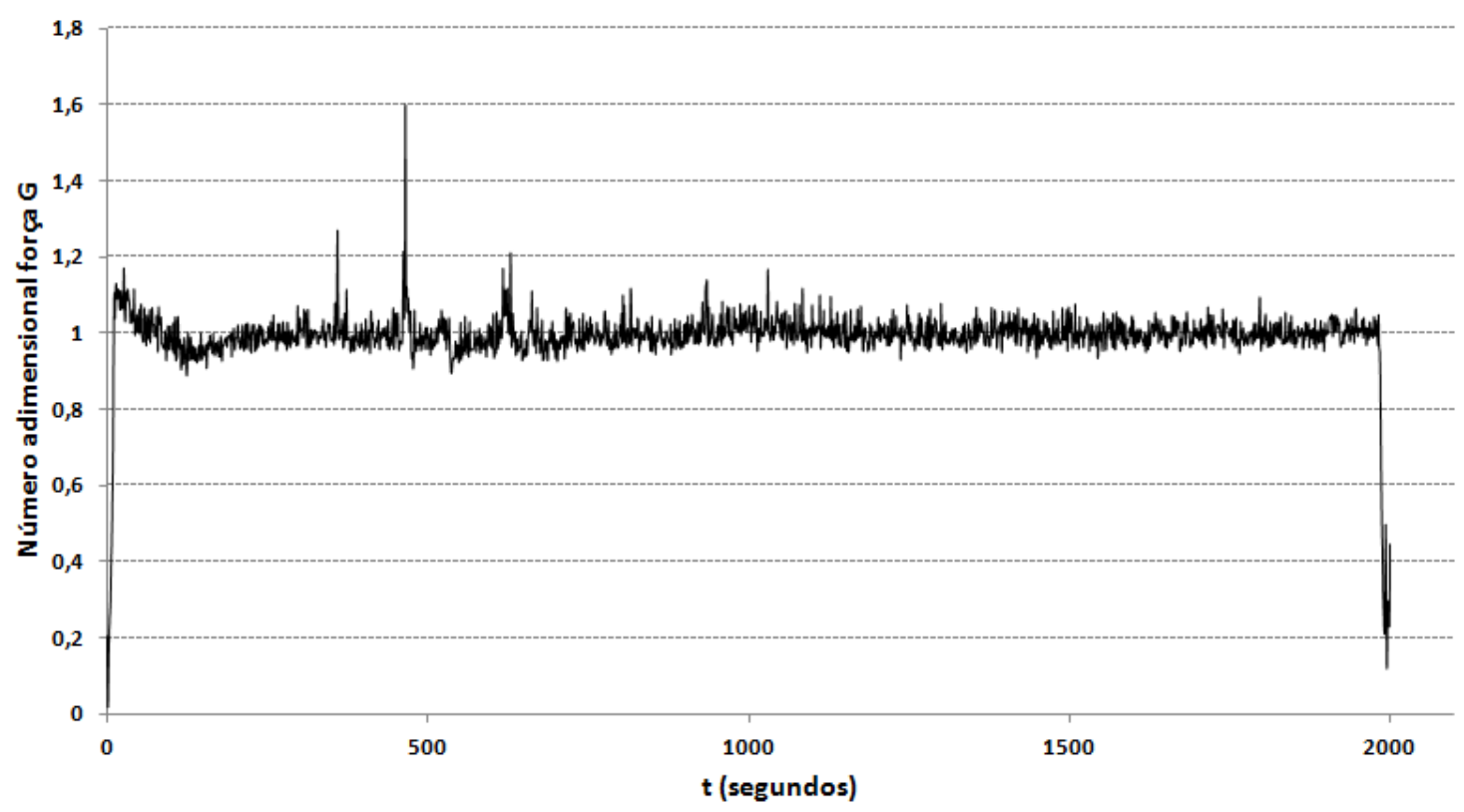

Figura 6 - Comportamento temporal da rotação dos motovibradores

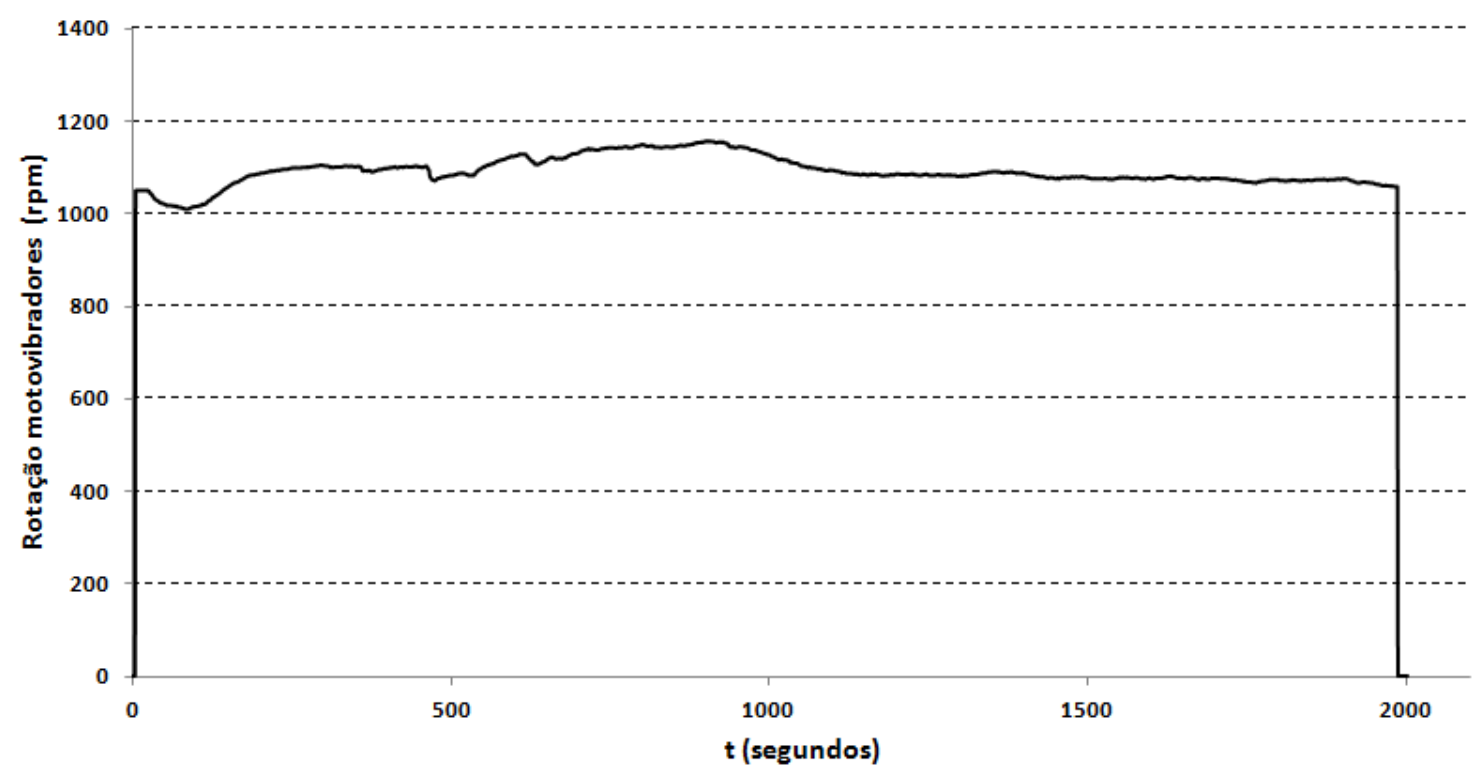

Entre os instantes $\mathrm{t}=88$ segundos e $\mathrm{t}=$ 909 segundos, ocorreu a atuação do sistema de controle proporcionando um aumento gradual na intensidade do sinal de saída enviado aos motovibradores, de maneira a manter o número adimensional da força-g constante. Após o instante $\mathrm{t}=909$ segundos, o sistema de controle voltou a diminuir a intensidade de correção, muito provavelmente em função da maior parte do material sólido contido na corrente de processo já ter sido alimentado à tela da peneira vibratória. Um aspecto importante na operação da bancada experimental foi a constatação experimental 
de que o material sólido dissolvido acabava por ser descarregado do tanque principal de alimentação logo no início da operação, havendo dificuldade em se manter a suspensão bem misturada ao longo de todo o período de realização do experimento.

$\mathrm{Na}$ Figura 7 é apresentado o comportamento temporal do número adimensional correspondente à força-g em resposta a variação em degrau de amplitude $1092 \mathrm{rpm}$ na frequência dos motores de acionamento dos motovibradores.

Figura 7 - Resposta do adimensional de força-g a uma variação em degrau aplicada na frequência dos motovibradores

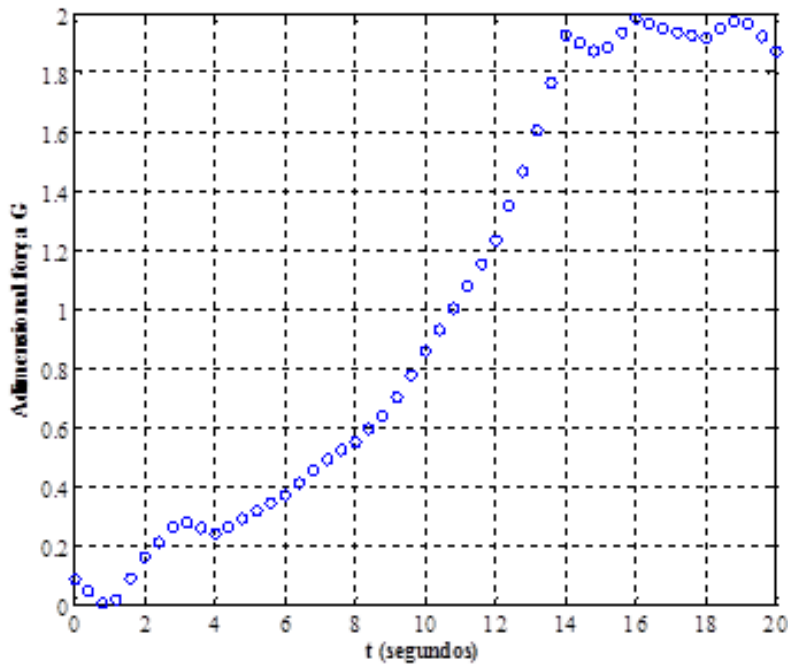

O comportamento temporal da variável de processo teor de umidade arrastado juntamente com os sólidos separados em função do número adimensional que caracteriza a força-g aplicada, para a peneira vibratória estudada e considerando a concentração volumétrica de sólidos de $1 \%$, é apresentado na Figura 8. Na Figura 9 é apresentado o diagrama de simulação correspondente (Garcia, 2005).
Figura 8-Resposta do teor de umidade nos sólidos separados à variação da amplitude do sinal do adimensional de força-g

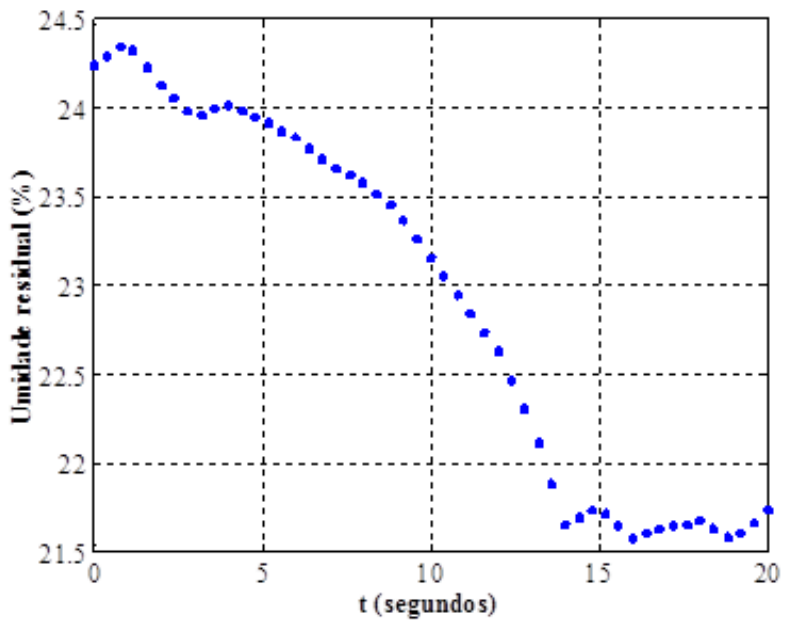

Com base nos resultados obtidos, verificou-se que à medida que o valor do adimensional de força-g aumentou, até alcançar a nova condição de estado estacionário, o teor de umidade residual diminuiu. Este comportamento era esperado, uma vez que a vibração deveria - de fato proporcionar uma maior remoção de liquido residual presente.

Figura 9- Diagrama de simulação do teor de umidade nos sólidos separados à variação da amplitude do sinal do adimensional de força-g Cv $=1 \%$

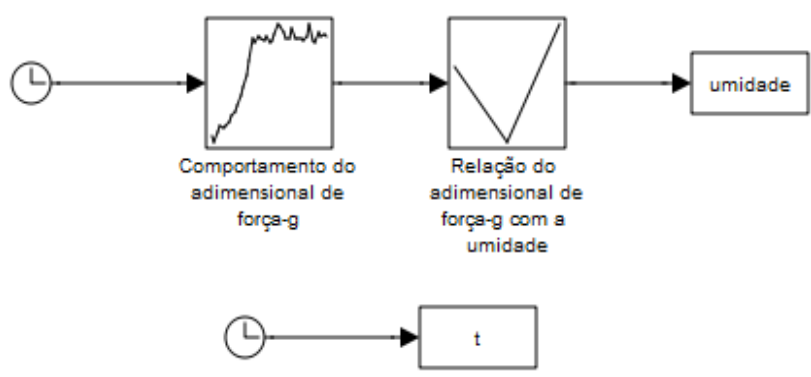

Considerando os resultados obtidos no experimento apresentado, pode-se concluir que o aumento da amplitude do adimensional da força-g contribuiu para diminuir o teor de umidade residual presente no material sólido retido sobre a tela da peneira para a 
concentração volumétrica da suspensão igual a $1 \%$. Pode-se, também, concluir que o sistema de controle e respectiva instrumentação empregados funcionaram de maneira coerente e adequada.

Com base neste modelo identificado, poder-se-á avaliar o impacto que alterações pré-estabelecidas no valor do número adimensional força-g teriam sobre o teor de umidade arrastado pelo material sólido granular retido sobre a tela da peneira.

O principal benefício que poderá resultar da aplicação do modelo no processo está relacionado ao melhor controle da operação, minimizando a perda de fluido de perfuração e maximizando a vida útil da tela da peneira.

Com base no aprendizado obtido neste trabalho, pretende-se desenvolver em etapa posterior o modelo matemático dinâmico identificado do comportamento do teor de umidade residual, com base em experimentos propostos futuramente especificamente para tal finalidade, os quais poderão ser usados como ferramenta de análise de processo.

Com base no modelo identificado, poder-se-á avaliar o impacto que alterações pré-estabelecidas no valor da força-g teriam sobre $\mathrm{o}$ teor de umidade arrastado pelo material sólido particulado retido sobre a tela da peneira.

Diante dos resultados apresentados, pode-se concluir que a peneira vibratória poderá ter o seu modelo matemático dinâmico identificado com base em experimentos propostos futuramente especificamente para tal finalidade, podendo este ser usado como ferramenta de analise de processo.

O principal benefício que poderá resultar da aplicação do modelo no processo está relacionado ao melhor controle da operação, minimizando a perda de fluido de perfuração o caso específico de poços $\mathrm{e}$ petróleo e maximizando a vida útil da tela da peneira.

\section{REFERÊNCIAS}

GARCIA, C. Modelagem e Simulação de Processos Industriais e de Sistemas Dinâmicos. 2005. EDUSP, São Paulo.

GUERREIRO, F. S., QUEIROZ, C. G., NEIRO, S. M. S., SÁ, C. H. M., GEDRAITE, R. (2013). Contribution to the study of dynamic behaviour of a shale shaker typically used in drilling fluid treatment unit. Anais do $V$ Encontro Nacional de Hidráulica de Poços de Petróleo e Gás, Teresópolis - RJ, 5 a 8 de agosto de 2013.

\section{LIU, T.; GAO, F.(2012). Industrial Process Identification and Control Design. Springer-Verlag, London - UK.}

LOBATO, F. S., OLIVEIRA-LOPES, L. C., GEDRAITE, R., NEIRO, S. M. S., MURATA, V. Identificação de modelos para unidades de recuperação do fluido de perfuração. In: IV Encontro Nacional de Hidráulica de Poços de Petróleo e Gás, Foz do Iguaçu - PR, 2011.

OGATA, K. Engenharia de Controle Moderno. Rio de Janeiro: Prentice Hall do Brasil, $3^{\text {a }}$ edição, 2000.

Raja, V., Chase, G. G., Jones, B. N. and Geehan, T. (2012). Computational Modeling and Experiments on Shale Shaker Performance. Proceedings of AADE Fluids Technical Conference and Exhibition. Houston, Texas.

RONAES, E., VIK, S. E., TAUGBOL, K., SJAHOLM, A.; KAAGESON-LOE, N. (2009). Remote real-time monitoring of particle size distribution in drilling fluids during drilling of a depleted HTHP reservoir. Proceedings of SPE/IADC, pp. 111, Manama, Bahrain, 26-28 October. 
SEBORG, D. E., EDGAR, T. F. and MELLINCHAMP, D. A. (2005). Process Dynamics and Control. Wiley, 2nd Ed., USA.

\section{AGRADECIMENTOS}

Os autores agradecem à UFU, ao CNPq e à PETROBRÁS pelos recursos concedidos e apoio em pesquisas no projeto referente ao termo de cooperação $\mathrm{N}^{\circ}$ 0050.0078502.12.9. 